Proceedings of the 7th Workshop on Quantum Chaos and Localisation Phenomena, May 29-31, 2015, Warsaw, Poland

\title{
Elastic Enhancement Factor: Mesoscopic Systems versus Macroscopic 2D Electromagnetic Analogue Devices
}

\author{
V.V. SOKOLOV ${ }^{a, b, *}$ AND O.V. ZHIROV ${ }^{a, c}$ \\ ${ }^{a}$ Budker Institute of Nuclear Physics of SB RAS, Novosibirsk, Russia \\ ${ }^{b}$ Novosibirsk Technical University, Novosibirsk, Russia \\ ${ }^{c}$ Novosibirsk State University, Novosibirsk, Russia
}

\begin{abstract}
Excess of probabilities of the elastic processes over the inelastic ones is a common feature of the resonance phenomena described in the framework of the random matrix theory. This effect is quantitatively characterized by the elastic enhancement factor $F^{(\beta)}$ that is the typical ratio of elastic and inelastic cross-sections. Being measured experimentally, this quantity can supply us with information on specific features of the dynamics of the intermediate complicated open system. We discuss properties of the enhancement factor in a wide scope from mesoscopic systems to macroscopic analogue electromagnetic resonators and demonstrate essential qualitative distinction between the elastic enhancement factor's peculiarities in these two cases. Complete analytical solution is found for the case of systems without time-reversal symmetry and only a few open equivalent scattering channels.
\end{abstract}

DOI: 10.12693 /APhysPolA.128.990

PACS: 05.45.Gg, 24.60.Lz, 05.45.Mt

\section{Introduction}

Enhancement of elastic processes as compared to inelastic ones is a remarkable phenomenon inherent in various resonance processes in nuclear and many-electron atomic physics as well as in analogue experiments with 2D open macroscopic devices. Various aspects of this effect have repeatedly attracted attention of theorists and experimentalist starting with the pioneering papers by Moldauer [1]. This subject has gained a solid foundation in the random matrix approach to the problem of the nuclear compound resonance scattering worked out in the fundamental paper [2]. On this ground, Verbaarschot [3] had managed to derive onefold integral representation of the factor $F^{(\beta=1)}$ (T-invariant systems) in the limit of very large number $M$ of very weak scattering channels (the Verbaarschot regime). In the case of $M$ equivalent channels with the transmission coefficients $T$ this factor depends on the only parameter $\eta=M T$. Later on, similar to $[2,3]$ calculations have been performed in the meaningful paper [4] for systems with broken timereversal symmetry $(\beta=2)$, which is relevant for the case of many-electron atoms in a strong magnetic field. Using the techniques developed in these papers one can show (see $[5,6])$ that in the Verbaarschot limit the enhancement factor can be represented in the following form:

$$
F^{(\beta)}(\eta)=1+\delta_{\beta 1}+\eta K^{(\beta)}(\eta),
$$

where the function $K^{(\beta)}(\eta)$ is the time delay variance,

$$
K^{(\beta)}=\frac{\left\langle Q^{2}\right\rangle}{\langle Q\rangle^{2}}-1=\int_{0}^{\infty} \mathrm{d} s \mathrm{e}^{-\eta s}\left[1-B_{2}^{(\beta)}(s)\right],
$$

while $B_{2}^{(\beta)}(s)$ stands for the Dyson spectral binary form factor belonging to the symmetry class $\beta$. As to the

\footnotetext{
*corresponding author; e-mail: V.V.Sokolov@inp.nsk.su
}

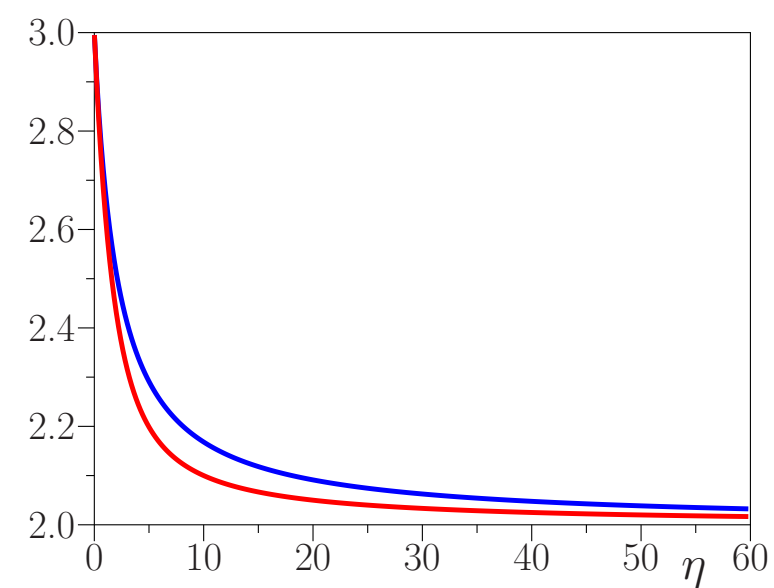

Fig. 1. $F^{(\beta)}$ versus $\eta$. Top curve $F^{(1)}(\eta)$, bottom $F^{(2)}(\eta)+1$.

parameter $\eta=M T$, it equals [5] to the ratio $\eta=t_{\mathrm{H}} / t_{\mathrm{W}}$ of two characteristic times: the Heisenberg time $t_{\mathrm{H}}=\frac{2 \pi}{d}$ defined by the mean level spacing $d$ of the Hermitian part $H$ of the total non-Hermitian effective Hamiltonian $\mathcal{H}$ describing the intermediate open system, and the dwell (or the Weisskopf) time $t_{\mathrm{W}}=\langle Q\rangle / T$ that is fixed by the resonance spectrum of $\mathcal{H}$. The binary form factors $B_{2}^{(\beta)}(s)$ are known for both the symmetry classes (see for example [7]). Moreover, in both these cases the $s$-integration can be fulfilled explicitly with the following results (illustrated in Fig. 1):

$$
F^{(2)}(\eta)=1+\frac{1-\mathrm{e}^{-\eta}}{\eta} .
$$

and

$$
\begin{aligned}
& F^{(1)}(\eta)=F^{(2)}(\eta)+1 \\
& \quad-\left(\frac{1+\eta / 2}{\eta} \mathrm{e}^{-\eta}-\frac{1-\eta / 2}{\eta}\right) \mathrm{e}^{\eta / 2} \operatorname{Ei}(-\eta / 2) .
\end{aligned}
$$


The enhancement factor decreases monotonically when the parameter $\eta$ grows and

$$
F^{(\beta)}(0)=2+\delta_{\beta 1} ; \quad F^{(\beta)}(\infty)=1+\delta_{\beta 1} .
$$

Being rather fast as long as the parameter $\eta$ remains relatively small, descent of the factors $F^{(\beta)}(\eta)$ slows subsequently down approaching gradually to their minimal values. At last, the slopes at the origin are identical for both symmetry classes:

$$
\left.\frac{\mathrm{d} F^{(\beta)}(\eta)}{\mathrm{d} \eta}\right|_{\eta=0}=-\frac{1}{2} \text {. }
$$

In spite of the apparent distinction between the expressions (3) and (4) their behavior is quite similar not only qualitatively but also quantitatively. Their difference remains within a few percent at most (see Fig. 2).

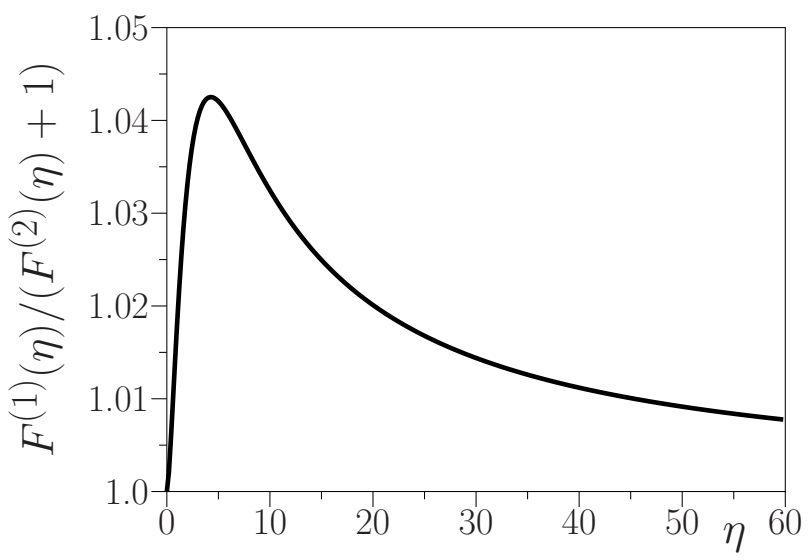

Fig. 2. The ratio $\frac{F^{(1)}}{F^{(2)}+1}$.

\section{From regular to chaotic dynamics}

In the mesoscopic systems discussed above, the chaotic behavior arises due to the residual interaction between quasi-particles moving in the common mean field. The intensity of this interaction, that is supposed to be controlled by some strength parameter $\kappa$, should be strong enough to ensure fully developed chaos. Otherwise, the dynamics acquires mixed character and can be described by a transient ensemble of Hamiltonians [8]. It has been shown in [5] that the relation (1) holds in the whole transition region. In the case of systems with broken time-reversal symmetry, the corresponding Dyson binary form factor has been found in [9]. This enables one to get analytical solution valid for any value of the interaction parameter (see [5]). As distinct from Eq. (3), the enhancement factor does not decreases monotonically with $\eta$ growing. Instead, at any fixed value of $\kappa$, the factor goes down at first (with the universal slope $-1 / 2$ at the origin $\eta=0$ ), reaches some minimal value and starts to grow approaching to the maximal value again. The depth and the position of the minimum depends on the interaction parameter $\kappa$, Fig. 3. In the limit $\kappa \rightarrow \infty$ the expression (3) recovers. Therefore, by measuring the enhancement factor experimentally we obtain information on the degree of chaoticity of the internal dynamics.

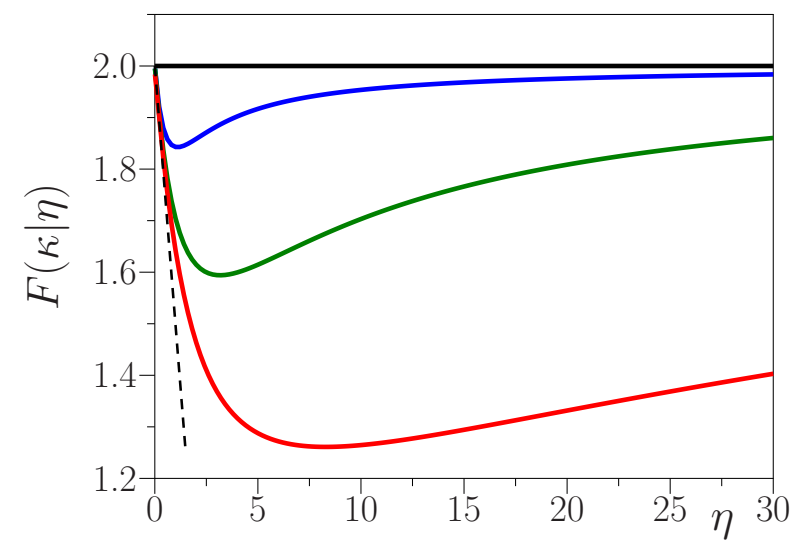

Fig. 3. $F^{(2)}(\eta \mid \kappa)$ for (from top to bottom) $\kappa=0$, $0.5,5,50$; dashed line indicates the slope at the point $\eta=0$

\section{Analogue experiments}

Many aspects of the theory of the quantum chaotic scattering can be analyzed and tested experimentally with the aid of macroscopic analogue devices. This method took on wide dissemination after pioneering experiments $[10,11]$ with open irregularly shaped $2 \mathrm{D}$ electromagnetic cavities (see for example $[12,13]$ and references therein). In particular, the elastic enhancement factor has thoroughly been measured for both symmetry classes as well as in the transient regime between them [13]. However, in contrast with the Verbaarschot regime of very large number of very weak channels the number of the channels is restricted in the analogue experiments to only a few ones, as a rule even to two. Under such conditions, the ruling in the Verbaarschot regime parameter $\eta$ becomes irrelevant and the enhancement factor depends on the number of channels and transmission coefficients separately. Supposing equivalence of the channels (see below), the enhancement factor looks as

$$
F_{M}^{(\beta)}(T)=1+\delta_{1 \beta}+(1-T) \frac{\boldsymbol{J}_{M}^{(\beta)}(T)}{\boldsymbol{P}_{M}^{(\beta)}(T)},
$$

where the functions $\boldsymbol{J}^{(\beta)}$ and $\boldsymbol{P}^{(\beta)}$ are given by the well known three- $(\beta=1)$ or twofold $(\beta=2)$ integrals $[2,4]$.

A general analytical formula valid for an arbitrary number of channels $M$ does not exist. Nevertheless, in the case of the systems with broken time-reversal symmetry, $(\beta=2)$, for any given number of channels the enhancement factor can be expressed explicitly as a ratio of two $(M-1)$-order polynomials. Dropping the subscript $\beta=2$, we get for example

$$
F_{2}(T)=\frac{4-T}{2+T}, \quad F_{4}(T)=3 \frac{8-6 T+4 T^{2}-T^{3}}{12+6 T-4 T^{2}+T^{3}}
$$

and so on. These and some more examples are illustrated in Fig. 4. It is seen that the larger the number of channels the faster enhancement factor decays when $T$ increases. Note that the established in Sect. 1 connection between the enhancement factor and delay time variance, that looks now as 


$$
K_{M}(T)=\frac{2}{T^{2}} \frac{1-(1-T)^{M+1}}{M^{2}-1},
$$

does not exist anymore [14].

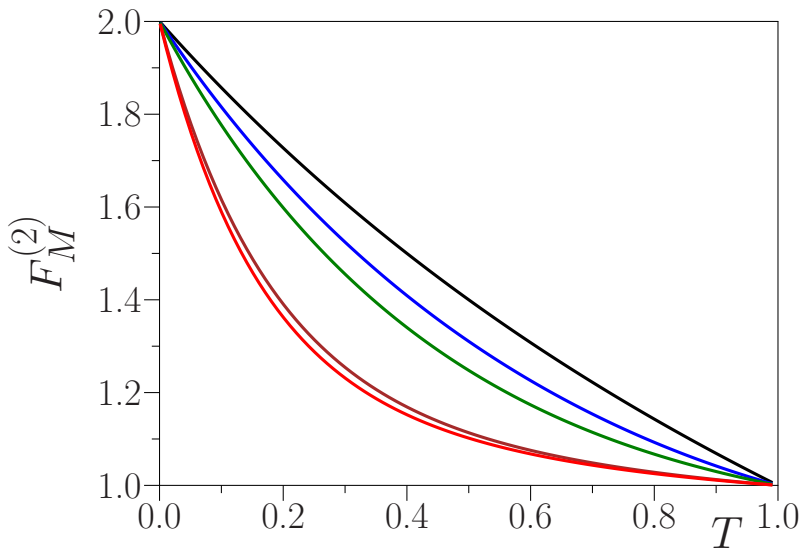

Fig. 4. $F_{M}$ versus $T$. From top to bottom $M=$ $2,3,4,9,10$.

\section{Two-channel model}

Below, we restrict ourselves to the practically most important case of only two open channels. Then the complete analytical treatment becomes possible for the systems without time-reversal symmetry. To begin with, let us analyse the practical significance of the equivalent channels assumption. To this end, suppose the opposite, $T_{1} \neq T_{2}$, and define two new variables:

$$
T=\frac{1}{2}\left(T_{1}+T_{2}\right), \quad \Delta=\frac{1}{2}\left(T_{1}-T_{2}\right),
$$

so that $T_{1}=T+\Delta, T_{2}=T-\Delta$ whereas $0<T<1$, $-\frac{1}{2}<\Delta<\frac{1}{2}$.

Though an explicit analytical solution exists even in this case the appropriate expression is extremely lengthy. Therefore we skip it and rather illustrate the result diagrammatically (see Fig. 5).

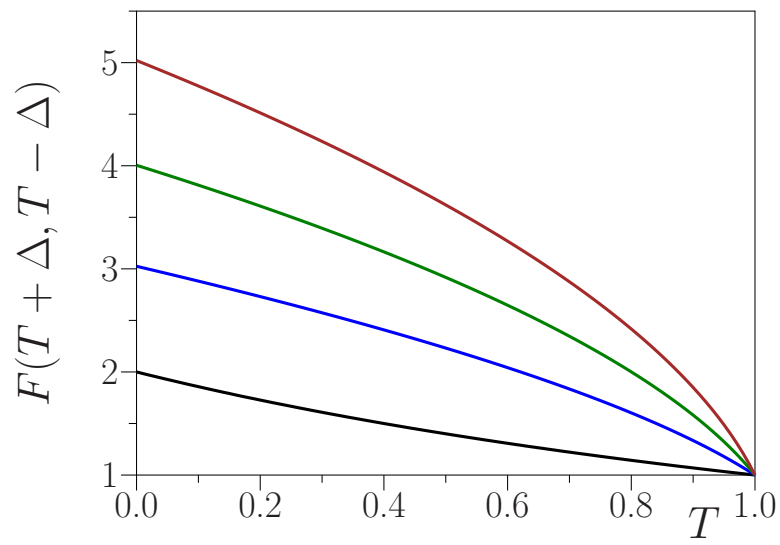

Fig. 5. $F(T+\Delta, T-\Delta)$ at different values of $\Delta$ : from bottom to top $\Delta=0,0.994 \mathrm{~T}, 0.99985 \mathrm{~T}, 0.9999985 \mathrm{~T}$ (analytical solution).
As long as $\Delta$ is noticeably smaller than $T$, the result is the same as in the case of equivalent channels and this approximation works quite well. Only when $\Delta$ is very close to $T$ enhancement factor can become arbitrarily large. The reason is quite clear: if one out of the two channels is almost closed everything is going on via the second one.

\section{The ohmic losses}

In the analogue experiments discussed here the ohmic losses always play an important role and cannot be neglected. The reasonable way to take them into account has been suggested in [4] and consists in introducing the overall decaying factor $\mathrm{e}^{-\gamma \tau}$. Analytical solution is still possible in the case $\beta=2$ and looks as

$$
F_{2}^{(\beta=2)}(T, \gamma)=1+(1-T) \frac{N(T, \gamma)}{D(T, \gamma)},
$$

where the functions $N$ and $D$ are found to be

$$
N(T, \gamma)=T\left(2 T^{2}-T \gamma+\gamma^{2}\right)+\gamma^{3} \mathrm{e}^{\gamma / T} \operatorname{Ei}(-\gamma / T),
$$
and

$$
\begin{aligned}
& D(T, \gamma)=T\left[T^{3}+\gamma^{2}-T \gamma(1+\gamma)+2 T^{2}\right. \\
& \left.\quad \times\left(2(\gamma-1)+\frac{3 \gamma}{\mathrm{e}^{\gamma}-1}\right)\right]+\gamma\left\{(1-T) \gamma^{2}\right. \\
& \left.-3 T^{2}[2-\gamma \operatorname{coth}(\gamma / T)]\right\} \mathrm{e}^{\gamma / T} \operatorname{Ei}(-\gamma / T) .
\end{aligned}
$$

This result is illustrated in the lower part of Fig. 6. At the point $T=0$, the enhancement factor drops vertically down to the value

$$
F(T=0, \gamma)=1+\frac{2}{\gamma\left(1+\operatorname{Coth}\left[\frac{\gamma}{2}\right]\right)}
$$

and, after that, approaches to its minimal value $F(T=$ $1, \gamma)=1$ almost horizontally.

No explicit analytical formulae can be derived in the case of $\beta=1$. Therefore we calculated the factor $F_{(M=2)}^{(\beta=1)}(T, \gamma)$ numerically to be able to compare the influence of the absorption in these two cases. The results are presented in Fig. 6. The interval $5.2<\gamma<7.4$

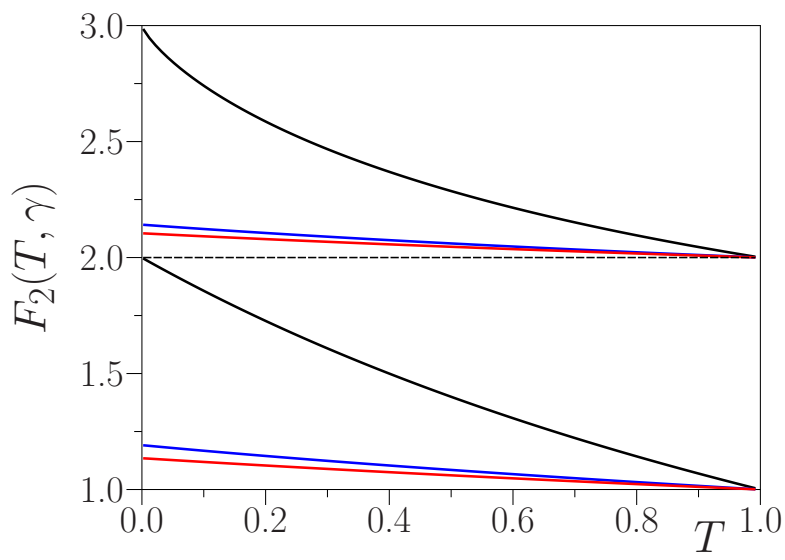

Fig. 6. $F_{2}^{(\beta)}(T, \gamma)$ for $\beta=1$ (up) and $\beta=2$ (down): $\gamma=0,5.2,7.4$ (from top to bottom). 
of absorption coefficients is the same as in the recent paper [15] reporting the results of experimental investigation of the elastic enhancement factor with the aid of analogue 2D electromagnetic resonators. It is clearly seen that the $T$-invariant systems is somewhat more sensitive to the influence of absorption. On the whole, the parasitic absorption washes away a considerable part of the information that is hidden in the dependence on the transmission coefficient.

\section{Summary}

In this paper, we focus on the specific features of the elastic enhancement factor depending on peculiarities of the chaotic open system one is dealing with. On the whole, this factor depends on the number $M$ of scattering channels as well as the channel's transmission coefficients. However, when the number of channels is very large, which is typical for example of the processes like the resonance nuclear reactions, the enhancement factor is controlled by the only parameter $\eta=M T$ that changes in very wide bounds (the Verbaarschot regime). Quite opposite situation takes place in the analogue experiments with 2D irregularly shaped billiards that are used for imitation of the quantum chaos. In the experiments of such a kind the number of channels is very restricted. As a result, the enhancement factor depends on the number of channels and on transmission coefficients separately. We have juxtaposed in detail the two specified regimes. We have succeeded in finding complete analytical solution valid for any fixed number $M$ of equivalent channels with arbitrary transmission coefficients $0<T<1$ in the case of systems without timereversal symmetry. More than that, in the practically significant case $M=2$ the influence of absorption has also received an explicit analytical description.

\section{Acknowledgments}

We are very grateful to L. Sirko for his interest in this work and significant critical remarks. V. Sokolov also appreciates useful discussions with J. Verbaarschot and F.M. Izrailev during the workshop on non-Hermitian Random Matrices "50 Years After Ginibre" (Israel, 2227 October 2014). This work is supported by the Ministry of Education and Science of the Russian Federation and by the Russian Foundation for Basic Research (grant 14-02-00424). At last, we greatly appreciate the countenance by the RAS Joint Scientific Program "Nonlinear Dynamics and Solitons".

\section{References}

[1] P.A. Moldauer, Phys. Rev. 123, 968 (1961), Phys. Rev. B 135, 642 (1964).

[2] J.J.M. Verbaarschot, H.A. Weidenmüller, M.R. Zirnbauer, Phys. Rep. 129, 367 (1985).

[3] J.J.M. Verbaarschot, Ann. Phys. 168, 368 (1985).

[4] Y.V. Fyodorov, D.V. Savin, H.-J. Sommers, J. Phys. A Math. Gen. 38, 10731 (2005).

[5] Y.A. Kharkov, V.V. Sokolov, Phys. Lett. B 718, 1562 (2013).

[6] N. Lehmann, D.V. Savin, V.V. Sokolov, H.-J. Sommers, Physica D 86, 572 (1995).

[7] M.L. Mehta, Random Matrices, 3rd ed., Elsevier, Amsterdam 2004.

[8] A. Pandey, Chaos Solitons Fractals 5, 1275 (1995); T. Guhr, Phys. Rev. Lett. 76, 2258 (1996); T. Guhr, A. Müller-Groeling, J. Math. Phys. 38, 1870 (1997).

[9] H. Kunz, B. Shapiro, Phys. Rev. E 58, 400 (1998).

[10] H.-J. Stöckmann, J. Stein, Phys. Rev. Lett. 64, 2215 (1990).

[11] S. Sridhar, Phys. Rev. Lett. 67, 785 (1991).

[12] Z. Pluhar, H.A. Weidenmüller, J.A. Zuk, C.H. Lewenkopf, F.J. Wegner, Ann. Phys. 243, 1 (1995); M. Lawniczak, S. Bauch, O. Hul, L. Sirko, Phys. Rev. E 81, 046204 (2010); M. Lawniczak, Sz. Bauch, O. Hul, L. Sirko, Phys. Scr. T143, 014014 (2011); M. Lawniczak, Sz. Bauch, O. Hul, L. Sirko, Phys. Scr. T147, 014018 (2012); M. Lawniczak, A. Nicolau-Kuklinska, S. Bauch, O. Hul, L. Sirko, in: Plenary talk at the Int. Conf. CHAOS2013, Istanbul (Turkey), 2013.

[13] B. Dietz, T. Friedrich, H.L. Harney, M. Miski-Oglu, A. Richter, F. Schafer, H.A. Weidenmuller, Phys. Rev. E 81, 036205 (2010).

[14] Y.V. Fyodorov, D.V. Savin, H.-J. Sommers, Phys. Rev. E 55, R4857 (1997).

[15] M. Lawniczak, M. Bialous, V. Yunko, Sz. Bauch, L. Sirko, Phys. Rev. E 91, 032925 (2015). 